\title{
KICKING ABILITY AND KICKING DEFICIT IN YOUNG ELITE SOCCER PLAYERS
}

\author{
Lois Rodríguez-Lorenzo, Miguel Fernández-Del Olmo, \\ José Andrés Sánchez-Molina, and Rafael Martín-Acero
}

Learning and Human Movement Control Group, University of A Coruña, A Coruña, Spain

\author{
Original scientific paper \\ https://doi.org/10.26582/k.50.2.2 \\ UDC: $796.012 .57: 796.332$
}

\begin{abstract}
:
Kicking ability in soccer has been evaluated predominantly by the maximum ball velocity. The kicking deficit (KD) may be defined as the percentage of the difference between the maximum ball velocity each player achieved by the non-preferred leg kick in relation to the preferred leg kick. This study aimed to compare: side-to-side kicking velocity, vertical jumps and anthropometrics between the younger (G-14) and older $(\mathrm{G}+14)$ than 14 years soccer players. An additional aim was to investigate associations among these variables. Participants were 92 young elite soccer players from the development programme of a top Spanish division club. They were divided into two age groups according to their growth and motor development stages $(10.80-13.55$ years, $\mathrm{n}=46 ; 14.02-16.39$ years, $\mathrm{n}=46)$. Student's $t$-test showed that the maximum kicking velocity with the preferred and non-preferred leg, jumping performance, and all anthropometric measurements were significantly $(p<.01)$ higher in $\mathrm{G}+14$ compared with G-14. In contrast, KD values remained stable $(15.31 \%$ $15.83 \%$ ) without significant differences between the groups. Pearson's correlation analysis revealed that vertical jump tests and anthropometric measurements correlated with kicking ball velocity produced by the preferred and non-preferred leg. Our results demonstrate that kicking skills are still not consolidated and power factors may be determinant for kicking performance in young soccer players. In addition, the KD may be a constant element for both the under and above 14 years soccer players and could result from an unequal and greater use of the preferred leg in comparison with the non-preferred leg.
\end{abstract}

Key words: ball velocity, skill, performance, jumping, non-preferred leg, strength

\section{Introduction}

Kicking ability represents the most important soccer-specific skill (Bacvarevic, et al., 2012) since it is used not only in passes, crosses, and clearances, but also to score goals, thus increasing the chances of winning games. In fact, an analysis of the 2010 Soccer World Cup revealed that $80.69 \%$ of the goals were achieved by kicking (Njororai, 2013). Moreover, although accuracy is an important factor, kicking performance in soccer has been evaluated predominantly via the maximum ball velocity (Markovic, Dizdar, \& Jaric, 2006). It is important for players to achieve a high ball velocity in soccer goal kicking to give the opposing goalkeeper less time to react (Dörge, Andersen, Sørensen, \& Simonsen, 2002). In addition, the ability to kick equally with both the preferred and non-preferred leg leads to an advantage for soccer players (Grouios, Kollias, Tsorbatzoudis, \& Alexandris, 2002), since it increases the number of scoring opportunities (Lago-Peñas \& Lago-Ballesteros, 2011). Therefore, improving the maximal kicking velocity with both the preferred and non- preferred leg must be an important objective for soccer coaches. Furthermore, the rationale for training both legs equally is to minimise potential asymmetry of forces acting on joints, to reduce muscle imbalances and decrease the workload on the preferred leg, which may eventually lead to overuse injuries (Hides, Stanton, Stanton, McMahon, \& Wilson, 2008). Another reason for the non-preferred leg to be trained and get strong enough in soccer players is to support coordinative motor actions of the preferred leg (Rahnama, Lees, \& Bambaecichi, 2015; Ruas, Brown, \& Pinto, 2015).

Despite the importance of the ability to kick with both the preferred and non-preferred leg in soccer, there is an agreement across studies that ball velocity is significantly higher after a kick performed with the preferred compared with the non-preferred leg (Barfield, Kirkendall, \& Yu, 2002; Dörge, et al., 2002). This pointed to a kicking deficit (KD) in the non-preferred leg, which could be in a range of 9.32-18.35\% of the kicking ball velocity with the preferred leg, depending on the sample features (Rodríguez-Lorenzo, Fernandez- 
del-Olmo, \& Martín-Acero, 2015). The KD was calculated as the average velocity values of the total sample rather than as individual values (RodríguezLorenzo, et al., 2015). Nonetheless, one recent study reported the individual values of the KD $(12.49 \pm 3.24 \%)$ and defined it as the percentage of the difference between the maximal ball velocity each player achieved by the non-preferred leg kick in relation to the preferred leg kick (RodríguezLorenzo, Fernandez-del-Olmo, Sánchez-Molina, \& Martín-Acero, 2016). Interestingly, in this study it was found that KD results were independent of the absolute maximal kicking ball velocity achieved by the preferred leg. Therefore, in addition to the absolute maximal ball velocity values, the KD might also be a helpful index when evaluating and monitoring individual capacity of each player to kick equally with the preferred and non-preferred leg.

Although there are several factors that may affect maximal kicking ball velocity, such as technical (Juárez, López, \& Navarro, 2010; Nunome, Lake, Georgakis, \& Stergioulas, 2006), biomechanical (Young \& Rath, 2011), physiological (Ferraz, Van Den Tillaar, \& Marques, 2012) and muscle strength factors (Masuda, Kikuhara, Demura, Katsuta, \& Yamanaka, 2005), Rodríguez-Lorenzo, et al. (2016) suggested that KD could result from the difficulty of exhibiting the same neuromuscular skills that occur during kicks with the non-preferred and preferred leg. This supports the significant relationship found between the height achieved in the vertical jump tests (SJ, CMJ, CMJA, 1RJA) and the maximum kicking velocity with the preferred leg, but not with the non-preferred one. However, this study has been conducted with adults and, thus, it remains unknown whether KD already exists in young soccer players and if it is associated with neuromuscular skills, as previously shown in adults. Indeed, ball velocity increments associated with age are likely to occur not only due to the skill development of the kicking pattern, but also due to the increased absolute muscle strength associated with growth and maturation (Poulmedis, Rondoyannis, Mitsou, \& Tsarouchas, 1988; Tol, Slim, van Soest, \& van Dijk, 2002; Wong, Chamari, Dellal, $\&$ Wisloff, 2009).

Therefore, as mentioned previously, the purpose of this study was to compare side-to-side kicking velocity, vertical jumps and anthropometrics between young soccer players under 14 years of age (G-14) and those over the age of 14 years $(\mathrm{G}+14)$. Moreover, this research aimed at investigating associations between these variables.

\section{Methods}

\section{Subjects}

Ninety-two elite male soccer players, belonging to the youth development programme of the Real
Club Deportivo de la Coruña, were tested as a part of their athletic training programme during the final month of the 2014/2015 competitive season (May 2014). The sample was divided into two subgroups according to their chronological age (players younger and players older than 14 years of age).

Groups G-14 (under 14 years) included 46 players (age: $12.18 \pm 0.94$ years; body mass: $40.86 \pm 7.84 \mathrm{~kg}$; body height: $149.60 \pm 9.42 \mathrm{~cm}$; body mass index: $18.09 \pm 1.65 \mathrm{~kg} \cdot \mathrm{m}^{-2}$; goalkeepers: $\mathrm{n}=6$, defenders: $n=16$, midfielders: $n=10$, and attackers: $\mathrm{n}=14)$. They had an average of $4.84 \pm 1.17$ years of soccer training experience, trained on average 2.5 times a week, and played one game per week during the last four years. Thirty-three players preferred to kick using their right leg, whereas thirteen preferred their left leg.

Group G+14 (above 14 years) included 46 players (age: $15.04 \pm 0.80$ years; body mass: $60.72 \pm 9.59 \mathrm{~kg}$; body height: $169.34 \pm 7.86 \mathrm{~cm}$; body mass index: $21.09 \pm 1.86 \mathrm{~kg} \cdot \mathrm{m}^{-2}$; goalkeepers: $\mathrm{n}=6$, defenders: $n=16$, midfielders: $n=12$, and attackers: $n=12$ ). They had an average of $7.82 \pm 0.79$ years of soccer training experience, trained on average 3.5 times a week, and played one game per week during the last four years. Thirty-four players preferred to kick using their right leg, whereas twelve preferred their left leg.

Experimental procedures of the study were carried out in accordance with the Spanish laws that regulated clinical research in humans (Royal Decree 561/1993), the Organic Law on Personal Data Protection 15/1999, and the necessary ethics principles in the 2013 review of the Declaration of Helsinki. The study was approved by the Ph.D. Committee of the Faculty of Sport Sciences and Physical Education of the University of A Coruña and by club authorities. After receiving a detailed explanation of the study's benefits and risks, all participants and their parents signed an informed consent form and completed a questionnaire giving personal, medical and training details. None of the subjects reported neurological diseases or recent injuries.

\section{Design and methods}

Participants familiarised themselves with the test procedures in four sessions, which were conducted once a week starting six weeks prior to the initial assessment in order to minimise any learning or habituation effects. Each familiarisation session consisted of the performance of the same experimental procedures for kicking and jumping tests. However, while in the experimental sessions these tests were carried out on different days, in these familiarisation sessions they were performed on the same week day. Participants had seven days of rest between the last familiarisation session and the first experimental day. 
Two experimental sessions were conducted on two days, separated by 48 hours of rest, in the atmosphere temperature of 16 to $18^{\circ} \mathrm{C}$. All tests were always performed in the same order due to the availability of sports facilities. However, this order should not be considered as a factor that affects the results, since the number of attempts was low and the rest time between sessions was 48 hours. The first experimental session started with the anthropometric measurements followed by the vertical jump tests, because the sports facility where these tests were made was only available at the beginning of the training week. The second experimental session consisted of the maximal kick test. The total duration of each experimental session was an hour and a half. To avoid inter-observer variability, the same experienced investigator tested all subjects.

\section{Testing procedures}

\section{Anthropometric measurements}

Eleven anthropometric measurements were recorded according to the International Society for the Advancement of Kinanthropometry's protocol (Marfell-Jones, Olds, Stewart, \& Carter, 2006). All anthropometric measurements were taken twice on the right side of the body by the same trained anthropometrist in a standardised order after a proper regulation of the measuring instruments.

Body mass was measured using a BFW300 platform scale (Adam Equipment Co. Ltd., UK) to the nearest $0.1 \mathrm{~kg}$. Body height was measured using a Harpenden stadiometer (Holtain Limited, UK) to the nearest $0.1 \mathrm{~cm}$. Body lengths and breadths were measured using a Siber-Hegner anthropometer (Zurich, Switzerland) to the nearest $0.1 \mathrm{~cm}$. Waist was measured using a Lufkin W606PM flexible tape (Cooper Industries, USA) to the nearest $0.1 \mathrm{~cm}$.

Anthropometric variables consisted of eleven measurements: body mass, body height, two lengths (trochanterion and tibiale laterale height), three girths (upper arm flexed and tensed girth; thigh girth; calf girth), and three breadths (biiliocristal; biepicondylar femur; ankle breadth). The mean value of two measurements at each body site was used for further analyses.

\section{Kicking test}

A modified version of the kick test described by Markovic et al. (2006) was used to measure maximal ball velocity. Foot preference was selfselected based on the players' response to the question with which foot they preferred to kick for the maximal ball velocity measurement. The kicking test was preceded by a standard 15-minute warm-up consisting of five minutes of indoor running at a self-selected pace, a 5-minute active stretching protocol for the lower limbs ( 2 sets $x 3$ repetitions of hip flexion and extension, hip abduction and adduction, knee extension and flexion, and ankle plan- tarflexion-dorsiflexion exercises), six submaximal and two maximal instep kicks performed by each leg. The active stretching protocol was included because it was imposed by the Medical Department of the Real Club Deportivo de la Coruña. All teams must follow the same warming-up procedure in order to prevent injuries, therefore its inclusion was respected during the development of the study.

After detailed explanations and a qualified demonstration, participants performed one practice trial with each leg. The following three trials with the preferred and the non-preferred leg were recorded as experimental trials. Participants were instructed to perform an instep kick of a stationary ball of standard size and standard inflation (Fédération Internationale de Football Association, FIFA, standard) as fast as possible towards the radar gun. To standardise the procedure, participants were restricted to a 5-step run-up from the position directly behind the ball. They were specifically instructed to focus only on achieving the maximum kicking ball velocity and the trials that missed the entire target area were repeated. Only six players needed to perform additional kicks and the maximum number of kicks performed by a player was eight. The order of testing was randomised between each leg. Each subject had at least 1-minute rest between two consecutive trials to avoid fatigue.

Kicking performance was assessed from maximal ball velocity. Velocity, expressed in $\mathrm{m} / \mathrm{s}$, was registered using a stationary Doppler radar gun (Stalker Sport 2, Stalker Radar, Plano, Texas, USA) that can measure speeds between $2.23 \mathrm{~m} \cdot \mathrm{s}^{-1}$ and $67.04 \mathrm{~m} \cdot \mathrm{s}^{-1}$ with accuracy of $\pm 0.045 \mathrm{~m} \cdot \mathrm{s}^{-1}$. The radar gun (operating frequency of $24.125 \mathrm{GHz}$ ) was attached to a $0.7 \mathrm{~m}$ high stand and positioned behind a net, approximately five $\mathrm{m}$ from the starting position of the ball. The size of the net was sufficient to cover all kicks that deviated less than $15^{\circ}$ from the direction of the radar gun (Markovic, et al., 2006). The radar gun was consistently calibrated immediately before the sessions according to the instructions given in the user's manual (Campo, Vaeyens, Philippaerts, Redondo, de Benito, \& Cuadrado, 2009).

The highest velocities of both the preferred and non-preferred legs were selected for further analysis. The coefficient of variation (CV) from the three trials was calculated. In addition, the KD variable was calculated according to the following formula (Rodríguez-Lorenzo, et al., 2016):

$$
\text { KD }=\left(\frac{\text { KVdomMax }- \text { KVnodommax }}{\text { KVdomMax }}\right) \cdot 100
$$

where KVdomMax and KVnodommax are the maximal velocities achieved using the preferred and non-preferred leg, respectively. 


\section{Vertical jump tests}

Before the vertical jump tests, the athletes completed 15 minutes of a standard warm-up which consisted of 5-minute indoor running at a self-selected pace, five minutes of the same active stretching protocol described in the section Kicking test, two submaximal squat jumps and two submaximal countermovement jumps. After the warmup, subjects were taught how to carry out each of the tests and performed one practice trial. The following two trials were recorded as experimental trials. Each subject had at least three minutes of rest between two consecutive trials of the vertical jumps in order to avoid fatigue.

The squat jump (SJ) and countermovement jump (CMJ) tests were performed according to the protocols described by Bosco, Luhtanen, and Komi (1983). In the SJs, the participants were instructed to maintain a static semi squatted position (knee angle around $60-90^{\circ}$, after individual motor adjustment) for two seconds before starting the jump without any preliminary movement. Individual ground reaction force traces were checked to verify that a countermovement had not occurred, and the trials in which a countermovement was detected were considered not valid and were repeated. For the CMJs, the subjects started from an upright standing position, performed a rapid downward movement (knee angle around $60-90^{\circ}$ ) followed by a dynamic complete extension of the lower limb joints. During these tests, starting position was with the feet parallel, shoulder width apart, while hands were kept on hips throughout the test.

The countermovement jump with arm swing (CMJA) and a reactive jump with arm swing (1RJA) tests were performed according to the protocols described by Acero, Sánchez, and Fernández-delOlmo (2012). In CMJA, participants performed the same movement as in the CMJ, but now with an arm swing movement. In the starting position, the arms were raised at shoulder level, flexed $90^{\circ}$ at elbows. When participants performed a rapid downward movement, arms simultaneously moved down with the elbows extended. When the lower limb joints were extended, the elbows began to flex and the hands went up to the face height. Finally, when the maximum height of the jump was achieved, the shoulders and elbows were locked in place. For the 1RJA, participants started from an upright standing position with their feet parallel, shoulder width apart, and arms straight down to the side of the body. First, they performed 2-3 progressive twolegged hops with their knees straight and helping themselves with the arm swing. Then, they jumped progressively ever higher during 5-6 seconds in order to achieve 2-3 maximum height jumps. After each of the jumps, the landing was made with the extended feet and knees, as in the take-off position.
All vertical jumps were performed on a force plate (Quattro Jump, Kisler, Winterhur, UK) in the following order: SJ, CMJ, CMJA and 1RJA. The vertical jump height was calculated according to the following formula (Bosco, et al., 1983):

$$
\text { SJ_H }=0.5 \cdot g \cdot\left(\frac{\text { flight time }}{2}\right)^{2}
$$

where flight time was the time between a take-off and landing. The greatest heights across all jump tests was considered for further analysis

\section{Statistical analysis}

Normality and homoscedasticity assumptions were checked respectively with Shapiro-Wilk and Levene's tests. A Student's $t$-test for independent samples with the Bonferroni correction was applied to compare anthropometric and vertical jump variables between $\mathrm{G}-14$ and $\mathrm{G}+14$ players.

We conducted an ANOVA of repeated measurements with one intrasubject factor (leg) and one intersubject factor (group) over the maximal kicking ball velocity and over the within-subject $\mathrm{CV}$ of the kicking velocity. Post-hoc analysis was performed with $t$-test and Bonferroni corrections. Effect size was evaluated with $\eta 2$ (partial eta-squared).

Pearson product correlation analysis was performed to assess the relationships between the kick test (KVdomMax, KVnodomMax), the anthropometric measurements and the vertical jumps (SJ, CMJ, CMJA, RJ). All statistical analyses were performed using SPSS version 21 (SPSS, Chicago, IL). A $p$ value $<.05$ was considered statistically significant.

\section{Results}

Normality assumption was not violated. Table 1 shows the results of the $t$-test to display the mean data differences between the groups (G-14 and $\mathrm{G}+14)$ for the anthropometric and vertical jump variables. The jumping performance and all the anthropometric measurements showed significantly larger values in group $\mathrm{G}+14$ than in group $\mathrm{G}-14$. The mean $( \pm \mathrm{SD})$ data for maximal kicking performance and the within-subject $\mathrm{CV}$ average of the kicking velocity, with the preferred and non-preferred leg for each age group, are presented in Table 2.

The ANOVA of repeated measurements showed a main effect for Leg $(\mathrm{F}=365.02, \mathrm{p}<.05, \eta 2=0.82)$ and Group $(\mathrm{F}=103.4, \mathrm{p}<.05, \eta 2=0.56)$ over maximal kicking velocity. There was a significant Leg*Group interaction $(\mathrm{F}=5.68, \mathrm{p}<.05, \eta 2=0.06$.). Post-hoc analysis revealed that maximal kicking velocity with the preferred leg (KVdomMax) was significantly $(\mathrm{p}<.001)$ higher than with the non-preferred leg in both groups. Maximum kicking velocity with the preferred and non-preferred leg showed signifi- 
Table 1. Mean ( $\pm S D$ ) of anthropometrics, kicking deficit and jump variables between the younger (Group -14) and older (Group +14) than 14 years soccer players

\begin{tabular}{|c|c|c|c|c|c|}
\hline \multirow[b]{2}{*}{ Dimension } & \multicolumn{2}{|c|}{ Group $-14(n=46)$} & \multicolumn{2}{|c|}{ Group +14 $(n=46)$} & \multirow[b]{2}{*}{$t$ Value } \\
\hline & Mean & SD & Mean & SD & \\
\hline Age (years) & 12.18 & 0.94 & 15.04 & 0.80 & $-14.89^{* *}$ \\
\hline Body mass (kg) & 40.86 & 7.84 & 60.72 & 9.59 & $-10.31^{* *}$ \\
\hline Stature $(\mathrm{cm})$ & 149.60 & 9.42 & 169.34 & 7.86 & $-10.24^{\star *}$ \\
\hline Body mass index $\left(\mathrm{kg} / \mathrm{m}^{2}\right)$ & 18.09 & 1.65 & 21.04 & 1.92 & $-7.47^{* *}$ \\
\hline Trochanterion height $(\mathrm{cm})$ & 82.34 & 10.02 & 90.96 & 10.06 & $-3.89^{* *}$ \\
\hline Tibiale laterale height $(\mathrm{cm})$ & 38.56 & 3.17 & 43.13 & 3.00 & $-6.69^{\star \star}$ \\
\hline Arm flexed and tensed girth $(\mathrm{cm})$ & 23.17 & 2.07 & 28.40 & 5.62 & $-5.69^{* *}$ \\
\hline Thigh girth (cm) & 42.90 & 4.03 & 50.60 & 5.07 & $-7.64^{* *}$ \\
\hline Calf girth $(\mathrm{cm})$ & 30.34 & 2.68 & 35.46 & 2.99 & $-8.17^{\star *}$ \\
\hline Biiliocristal breadth (cm) & 23.65 & 1.64 & 26.70 & 1.64 & $-8.40^{* *}$ \\
\hline Biepicondylar femur breadth $(\mathrm{cm})$ & 9.28 & 0.70 & 10.02 & 1.01 & $-3.89^{* *}$ \\
\hline Ankle breadth (cm) & 6.52 & 0.58 & 6.90 & 0.43 & $-3.33^{\star *}$ \\
\hline Kicking Deficit & 15.31 & 7.32 & 15.83 & 7.88 & -0.32 \\
\hline $\mathrm{SJ}(\mathrm{cm})$ & 4.43 & 22.84 & 7.01 & -1.69 & $-14.89^{* *}$ \\
\hline $\mathrm{CMJ}(\mathrm{cm})$ & 22.59 & 4.76 & 28.32 & 5.07 & $-5.33^{* *}$ \\
\hline CMJA (cm) & 25.30 & 4.98 & 32.53 & 5.31 & $-6.43^{* *}$ \\
\hline RJ (cm) & 23.05 & 4.16 & 27.99 & 4.40 & $-5.29^{\star *}$ \\
\hline
\end{tabular}

Note. $\mathrm{SJ}=$ squat jump; $\mathrm{CMJ}=$ countermovement jump; $\mathrm{CMJA}=$ countermovement jump with arm swing; $1 \mathrm{RJA}=$ reactive jump with arm swing; statistical significance: ${ }^{*} p<.05,{ }^{* *} p<.01$

Table 2. Mean values $( \pm S D)$ of the maximum kicking velocity and within-subject $C V$ of the kicking velocity between younger $($ Group -14) and older $($ Group +14$)$ than 14 years soccer players

\begin{tabular}{lccccc}
\hline & \multicolumn{4}{c}{ Maximum velocity preferred leg } & \multicolumn{2}{c}{ Maximum velocity non-preferred leg } \\
\cline { 2 - 5 } & $\mathrm{m} / \mathrm{s}$ & $\mathrm{CV}$ & $\mathrm{m} / \mathrm{s}$ & $\mathrm{CV}$ & $\mathrm{p} \mathrm{value}^{1}$ \\
\hline Group -14 & $22.25 \pm 2.11$ & $5.10 \pm 3.69$ & $18.8 \pm 2.28$ & $5.94 \pm 3.33$ & $.001^{*}$ \\
Group +14 & $27.54 \pm 2.45$ & $3.94 \pm 2.02$ & $23.14 \pm 2.58$ & $5.67 \pm 3.50$ & $.001^{*}$ \\
p value $^{2}$ & $.001^{*}$ & & $.001^{*}$ & & \\
\hline
\end{tabular}

Note. ${ }^{*}$ Significant differences in the maximum kicking velocity between the legs ( $p$ value $\left.{ }^{1}\right)$ and groups $\left(p\right.$ value $\left.{ }^{2}\right)$

cantly $(\mathrm{p}<.001)$ larger values in group $\mathrm{G}+14$ than in group G-14.

The ANOVA of repeated measurements showed a main effect for $\operatorname{Leg}(\mathrm{F}=9.07, \mathrm{p}<.05, \eta 2=.10$.) over the within-subject $\mathrm{CV}$ of the kicking velocity. The within-subject CV values of the kicking velocity were not significantly affected by the group. No interactions between Leg and Group were found for the $\mathrm{CV}$ of the kicking. Post-hoc analysis revealed that the within-subject $\mathrm{CV}$ of the kicking velocity with the preferred leg was significantly $(p<.01)$ lower compared to the non-preferred leg.

Table 3 shows the coefficients of correlation among the variables. There was a significant high positive correlation between age, body mass, stature, and kicking ball velocity with the preferred and non-preferred leg in both age grups. A greater number of anthropometric measurements corre- lated with the preferred kicking velocity (20/22) compared to the non-preferred (16/22) for the total of the two groups. Maximum kicking velocities between the legs correlated significantly for both groups $(\mathrm{r}=.72, p<.01)(\mathrm{r}=.65, p<.01)$. The results obtained from the vertical jump tests revealed a significant and moderate-high relationship between the maximum kicking velocity with the preferred and non-preferred legs and the height of each vertical jump (SJ, CMJ, CMJA, RJ).

\section{Discussion and conclusions}

This study aimed to compare side-to-side kicking velocity, vertical jumps and anthropometrics between soccer players younger (G-14) and older $(\mathrm{G}+14)$ than 14 years. An additional aim was to investigate associations among these variables. The main results were that the maximum ball velocity 
Table 3. Correlation values between variables

\begin{tabular}{|c|c|c|c|c|}
\hline \multirow[t]{2}{*}{ Dimension } & \multicolumn{2}{|c|}{$\begin{array}{l}\text { Maximum velocity } \\
\text { preferred leg }\end{array}$} & \multicolumn{2}{|c|}{$\begin{array}{l}\text { Maximum velocity } \\
\text { non-preferred leg }\end{array}$} \\
\hline & G-14 & $G+14$ & G-14 & $\mathrm{G}+14$ \\
\hline Age (years) & $.689^{\star *}$ & $.632^{\star *}$ & $.525^{\star *}$ & $.335^{\star}$ \\
\hline \multicolumn{5}{|l|}{ Anthropometric measurements } \\
\hline Body mass $(\mathrm{cm})$ & $.691^{* *}$ & $.855^{\star \star}$ & $.502^{* *}$ & $.535^{\star *}$ \\
\hline Stature $(\mathrm{cm})$ & $.738^{* \star}$ & $.753^{\star \star}$ & $.574^{* *}$ & $.486^{* *}$ \\
\hline Body mass index $\left(\mathrm{kg} / \mathrm{m}^{2}\right)$ & $.374^{* *}$ & $.719^{\star *}$ & .210 & $.401^{*}$ \\
\hline Trochanterion height $(\mathrm{cm})$ & .101 & $.335^{*}$ & .198 & .047 \\
\hline Tibiale laterale height $(\mathrm{cm})$ & $.684^{* \star}$ & .306 & $.565^{* *}$ & .222 \\
\hline Arm flexed and tensed girth $(\mathrm{cm})$ & $.605^{\star \star}$ & $.527^{\star \star}$ & $.357^{*}$ & $.419^{* *}$ \\
\hline Thigh girth $(\mathrm{cm})$ & $.599^{* *}$ & $.626^{* *}$ & $.353^{*}$ & .284 \\
\hline Calf girth (cm) & $.599^{* *}$ & $.727^{* *}$ & $.465^{* *}$ & $.527^{* *}$ \\
\hline Biiliocristal breadth (cm) & $.645^{\star *}$ & $.587^{* *}$ & $.470^{* *}$ & .221 \\
\hline Biepicondylar femur breadth (cm) & $.385^{*}$ & $.500^{* *}$ & .264 & .260 \\
\hline Ankle breadth $(\mathrm{cm})$ & $.493^{\star *}$ & $.476^{* *}$ & $.298^{*}$ & $.365^{*}$ \\
\hline \multicolumn{5}{|l|}{ Kicking test } \\
\hline Maximun velocity preferred leg & - & - & $.723^{* *}$ & $.650^{* *}$ \\
\hline Maximun velocity non-preferred leg & $.723^{* *}$ & $.650^{* *}$ & - & - \\
\hline Kicking deficit & .108 & .108 & .108 & .108 \\
\hline \multicolumn{5}{|l|}{ Jump test } \\
\hline $\mathrm{SJ}(\mathrm{cm})$ & $.569^{* *}$ & $.513^{* *}$ & $.455^{* *}$ & $.495^{* *}$ \\
\hline $\mathrm{CMJ}(\mathrm{cm})$ & $.576^{\star \star}$ & $.465^{\star *}$ & $.524^{* *}$ & $.446^{\star *}$ \\
\hline CMJA (cm) & $.665^{\star *}$ & $.616^{* *}$ & $.594^{* *}$ & $.606^{* *}$ \\
\hline $\mathrm{RJ}(\mathrm{cm})$ & $.302^{* *}$ & $.317^{* *}$ & $.505^{* *}$ & $.420^{* *}$ \\
\hline
\end{tabular}

Note. $\mathrm{SJ}=$ squat jump; $\mathrm{CMJ}=$ countermovement jump; $\mathrm{CMJA}=$ countermovement jump with arm swing; $1 \mathrm{RJA}=$ reactive jump with arm swing; ${ }^{*}$ correlations significant at $p<.05 ;{ }^{* *}$ correlations significant at $p<.01$.

after a soccer kick was, for the entire sample, significantly different between the preferred and nonpreferred leg, indicating a KD in the non-preferred leg. Furthermore, there were, for each group, significant correlations between the height achieved in the vertical jump tests (SJ, CMJ, CMJA and RJ) and the maximum kicking velocity with the preferred and non-preferred leg.

To the best of our knowledge, the present study is the first to report the individuals' values of the KD in young soccer players. Surprisingly, our results showed that KD is a constant element in young elite soccer players, since their values remained stable without significant changes between these age groups: $15.31 \pm 7.32 \%$ for G-14 and $15.83 \pm 7.88 \%$ for $\mathrm{G}+14$. The poorer kicking performance with the nonpreferred leg in elite players shown by the tests was in agreement with the results obtained in previous studies (Bacvarevic, et al., 2012; Dörge, et al., 2002; Nunome, et al., 2006). Those studies reported mean differences of $15.20 \%$ between the preferred and non-preferred legs in 106 youth Serbian national players divided into four age groups (12-15). A similar difference, more specifically of $15.58 \%$, was also reported (Nunome, et al., 2006) in highly skilled soccer players $(\mathrm{N}=5$, age $=16.8 \pm 0.4)$. Lower differences $(12 \%)$ were reported (Malý, Zahálka, Malá, \& Teplan, 2014) in youth Czech national team players $(\mathrm{n}=22$, age $=15.6 \pm 0.4$ years $)$ and in seven skilled soccer players (12.95\%) (Dörge, et al., 2002). However, these percentages were calculated from the total average values reported by those papers, and this did not allow reliable comparisons across different studies. Therefore, comparison of our findings to others must be done with caution.

Interestingly, our results show that despite no significant difference in KD values was found among age groups, the maximum kicking velocity with the preferred and non-preferred leg was significantly higher in $\mathrm{G}+14$. In addition, there was no correlation between KD and maximal kicking velocity with the preferred and non-preferred leg in either age group. Taken together, this discovery revealed that KD is independent of the absolute maximal kicking velocity of the player.

In order to explore the nature of $\mathrm{KD}$, we conducted a correlation analysis for each age group. We found a significant relationship between the 
height achieved in vertical jump tests (SJ, CMJ, CMJA, 1RJA) and the maximum kicking velocity with the preferred leg. These findings are consistent with those of Mercé, González, Mayo, Pardo and Sorli (2004) who reported a moderate significant relationship between kicking velocity with the preferred leg and the height reached in SJ $(\mathrm{r}=40)$ and $\mathrm{CMJ}(\mathrm{r}=.41)$ in young elite soccer players, the result that was similar to the values obtained in our study. This discovery agrees with those reported in adult elite soccer players, (Rodríguez-Lorenzo, et al., 2016) which described a moderate significant relationship between kicking velocity with the preferred leg and the height reached in SJ $(\mathrm{r}=.48)$, CMJ ( $\mathrm{r}=.58)$, CMJA $(\mathrm{r}=.44)$ and $\mathrm{RJ}(\mathrm{r}=.51)$ in adult elite male soccer players. Higher correlation $(\mathrm{r}=0.91)$ was found by Clark and Brooks (2011) with $\mathrm{CMJ}$ in adult elite female soccer players, which was in contrast to other studies that showed low significant relationships (Sousa, Garganta, \& Garganta, 2003) or no significant relationship (Juárez, et al., 2008, 2010) between SJ and CMJ with maximal kicking velocity with the preferred leg. Several methodological factors such as the use of different testing protocols and instruments, the moment of the season when the test was carried out, and the subjects' expertise level and age can help explaining these differences.

Interestingly, we also found a significant relationship between the maximum kicking velocity with the non-preferred leg and the height achieved in the vertical jump tests. In contrast to the preferred leg, the relationship between kicking velocity with the non-preferred leg and the vertical jump performance has received minimal research attention in the literature. To the best of our knowledge, only one study, conducted by our group, in elite adult players attempted to examine this relationship (Rodríguez-Lorenzo, et al., 2016), and found that this correlation was absent using exactly the same methodology as the present study. Nunome et al. (2006) attributed the differences in kick biomechanics between the two limbs to the skill level of the players (Nunome et al. 2006), i.e. the higher the skill level, the greater the importance of the coordination factors. Furthermore, Schmidt (1988) indicated that the expertise when accomplishing a task was related to lower variability in performance. Higher within-subject CV (5.16) values were found in the present study, compared to those reported in our previous study with adult soccer players (1.16). Also a lower maximal kicking performance was discovered. Those two facts indicate that kicking skill in young soccer players has not been consolidated yet. Moreover, although a correlation does not imply causality between variables, our results suggest that power factors might play an important role in kicking performance of young soccer players. However, in adult soccer players, high muscle specificity, developed due to a higher practice time accumulation (Shan, 2009) and a higher use of the preferred leg versus the non-preferred one (Oliva, et al., 2015) may lead to a better intersegmental pattern and transfer of velocity from the foot to the ball when kicking with the preferred leg (Dörge, et al., 2002). Indeed, during a soccer game, the use of the preferred leg occurs about $90 \%$ of the time in controls, passes, and crosses of the ball, and about $70 \%$ in kicks aimed at the goal (Oliva, et al., 2015). As a result, the soccer player develops a highly specific muscular function for each leg. While the non-preferred leg is trained to be activated in an isometric fashion (to provide a stable platform for the swing of the kicking leg), the preferred leg is characterised by an explosive action (Dörge, et al., 2002; Young \& Rath, 2011). This muscle specificity could be compromised during the kicking action with the non-preferred leg, which may explain the lack of correlation with the vertical jump in adult soccer players.

For instance, in both G-14 and G+14 groups, most of the anthropometric measurements were significantly associated with the maximal kicking velocities achieved with the preferred and nonpreferred leg. The present findings seem to be consistent with another research conducted in young soccer players, which found that maximal kicking velocity was significantly correlated with body mass, height and body mass index (Bekris, et al., 2015; Brahim, Bougatfa, \& Mohamed, 2013; Wong, et al., 2009). However, adult soccer players did not show any correlation between those parameters (Rodríguez-Lorenzo, et al., 2016). One possible explanation is that during the prepubertal and pubertal periods, transitory anthropometrical features associated with physical and maturity process, such as weight, height and strength (Figueiredo, Gonçalves, Coelho, Silva, \& Malina, 2009; Malina, et al., 2000, 2005; Ré, Cattuzzo, Santos, $\&$ Monteiro, 2014) may have a high influence on the kicking performance. However, after puberty, when the transitory differences associated with the maturity process cease to exist, the anthropometric variables have less importance in the performance. Accordingly, selecting young players based on their anthropometric characteristics could only provide short-term benefits in soccer team performance. Once players complete their physical growth, the anthropometric advantages decrease and technical and tactical factors become significantly more important (Ré, Corrêa, \& Boehme, 2010). Therefore, technical and tactical factors should be mainly considered in the selection process of young soccer players (Reilly, Williams, Nevill, \& Franks, 2000; Wong, et al., 2009).

Taking into account all the above-mentioned considerations, it seems that KD has a multifactorial nature and, thus, it can result from the influence of 
different factors, which correlate and influence each other, according to the experience of the player. This is of relevance to establish the best training procedures in order to reduce the KD. Our outcomes are in line with the result of other very recent studies (de Villarreal, Suarez-Arrones, Requena, Haff, \& Ferrete, 2015; Ramírez-Campillo et al., 2014, 2015) that demonstrated that the application of different strength training programmes (jumps and sprints, and plyometric exercises adapted to the age of our player) can increase ball velocity values in young soccer players. Furthermore, this kind of training should be incorporated in the daily workout routines since we can improve, at the same time, two of the most important and determinant soccer skills: jumping (heading) and kicking. However, it has been recommended that strength training should be integrated with other types of training involving the actual movement pattern to increase the performance within more complex movement patterns and in combination with regular soccer training (Aagaard, Simonsen, Trolle, Bangsbo, \& Klausen, 1996; Manolopoulos, Papadopoulos, \& Kellis, 2006). It has been proved that incorporating analytical tasks to improve kicking performance with the non-preferred leg increases the use of this leg by the player during competition (Guilherme, Garganta, Graça, \& Seabra, 2015). However, this action should not be limited to analytical tasks and coaches should also reward and give more reinforcing positive feedback to their players to continue using their nonpreferred leg in other parts of training sessions and matches, avoiding negative feedback when players fail to do so (Velázquez \& Jorge, 2012). These behaviours should be a determining factor for consolidating performance improvement with the non-preferred leg and gradually reducing KD in order to reach a point where players perform well with both the preferred and non-preferred leg and where they do not have to resort to safety situations all the time by using the preferred leg and, therefore, increasing the KD.

Our study has several limitations. The players were part of the youth development programme of a professional soccer team (Real Club Deportivo de la Coruña) that play in the best division of Spain (LFP). Therefore, extrapolation of our findings to other teams and soccer player levels must be done with caution. Furthermore, the subjects were tested during the final month of the competitive season and, thus, it remains to be explored whether our findings are consistent throughout the competitive season.

In summary, kicking deficit (KD) is a constant element in young elite soccer players, and its values remain stable without significant changes between $\mathrm{G}-14$ and $\mathrm{G}+14$ groups. The unequal and greater use of the preferred compared with the non-preferred leg in training sessions and games is one of the main factors that could affect asymmetric performance of the legs, since it produces a highly specific muscular function for each leg. Likewise, according to our results, we could suggest that, in young soccer players, the kicking skill is still not consolidated and the power factors may be determinant for the kicking performance.

Young players may need to perform a greater volume/intensity of resistance/explosive strength training to improve kicking velocity and jumping performance of both the preferred and non-preferred legs, as they present lower results than older players in those variables.

High kicking deficit between both the older and younger players may show that either this sideto-side asymmetry is a result from specific skills needed for soccer, or that strength and conditioning coaches should target a kicking velocity equivalency between legs for soccer players to improve their performance in the sport.

\section{References}

Aagaard, P., Simonsen, E., Trolle, M., Bangsbo, J., \& Klausen, K. (1996). Specificity of training velocity and training load on gains in isokinetic knee joint strength. Acta Physiologica Scandinavica, 156, 123-129.

Acero, R.M., Sánchez, J.A., \& Fernández-del-Olmo, M. (2012). Tests of vertical jump: Countermovement jump with arm swing and reaction jump with arm swing. Strength and Conditioning Journal, 34(6), 87-93.

Bacvarevic, B.B., Pazin, N., Bozic, P.R., Mirkov, D., Kukolj, M., \& Jaric, S. (2012). Evaluation of a composite test of kicking performance. Journal of Strength and Conditioning Research, 26(7), 1945-1952.

Barfield, W.R., Kirkendall, D.T., \& Yu, B. (2002). Kinematic instep kicking differences between elite female and male soccer players. Journal of Sports Science and Medicine, 1(3), 72-79.

Bekris, E., Gioldasis, A., Bekris, V., Gissis, I., Komsis, S., \& Mitrousis, I. (2015). The relationship of kicking ball velocity with anthropometric and physiological factors in soccer. Sport Science Review, 24(1-2), 71-87.

Bosco, C., Luhtanen, P., \& Komi, P.V. (1983), A simple method for measurement of mechanical power in jumping. European Journal of Applied Physiology, 50(2), 273-282. 
Brahim, M.B., Bougatfa, R., \& Mohamed, A. (2013). Anthropometric and physical characteristics of Tunisian young soccer players. Advances in Physical Education, 3(3), 125.

Campo, S., Vaeyens, R., Philippaerts, R., Redondo, J., de Benito, A., \& Cuadrado, G. (2009). Effects of lower-limb plyometric training on body composition, explosive strength, and kicking speed in female soccer players. Journal of Strength and Conditioning Research, 23(6), 1714-1722.

Clark, S., \& Brooks, K. (2011). Relationship between soccer specific skills and anthropometric data in NCAA Division I female soccer athletes. Journal of Strength and Conditioning Research, 25, S63-S64.

de Villarreal, E.S., Suarez-Arrones, L., Requena, B., Haff, G.G., \& Ferrete, C. (2015). Effects of plyometric and sprint training on physical and technical skill performance in adolescent soccer players. Journal of Strength and Conditioning Research, 29(7), 1894-1903.

Dörge, H.C., Andersen, T.B., Sørensen, H., \& Simonsen, E.B. (2002). Biomechanical differences in soccer kicking with the preferred and the non-preferred leg. Journal of Sports Sciences, 20(4), 293-299.

Ferraz, R., Van Den Tillaar, R., \& Marques, M.C. (2012). The effect of fatigue on kicking velocity in soccer players. Journal of Human Kinetics, 35(1), 97-107.

Figueiredo, A., Gonçalves, C., Coelho, J., Silva, J., \& Malina M. (2009). Characteristics of youth soccer players who drop out, persist or move up. Journal of Sports Science, 27(9), 883-891.

Grouios, G., Kollias, N., Tsorbatzoudis, H., \& Alexandris, K. (2002). Over-representation of mixed-footedness among professional and semi-professional soccer players: An innate superiority or a strategic advantage? Journal of Human Movement Studies, 42(1), 19-30.

Guilherme, J., Garganta, J., Graça, A., \& Seabra, A. (2015). Influence of non-preferred foot technical training in reducing lower limbs functional asymmetry among young football players. Journal of Sports Sciences, 33(17), 1790-1798.

Hides, T., Stanton, W., Stanton, P., McMahon, K., \& Wilson, S. (2012), Psoas and quadratus lumborum muscle asymmetry among elite Australian Football League players. British Journal of Sports Medicine, 44(8), 563-567.

Juárez, D., López, C., \& Navarro, E. (2010). Analysis of the soccer kick and its relationship with the vertical jump in young top-class soccer players. Revista Internacional de Ciencias del Deporte, 6(19), 29-41.

Juárez, D., Valdivielso, F.N., Rubio, R.M., Ravé, J.M., Blázquez, A.A., \& Fernández-Arroyo, V. M. (2008). Relationship among maximal strength in squat exercise, jump, sprint and kicking ball performance. Revista Internacional de Ciencias del Deporte, 4(10), 1-12.

Lago-Peñas, C., \& Lago-Ballesteros, J. (2011). Game location and team quality effects on performance profiles in professional soccer. Journal of Sports Science and Medicine, 10(3), 465-471.

Luhtanen, P. (1988). Kinematics and kinetics of maximal instep kicking in junior soccer players. In T. Reilly, A. Lees, K. Davids \& W. J. Murphy (Eds.), Science and football (pp. 441-448). New York: E\&F.N. Spon.

Malina, R.M., Cumming, S.P., Kontos, A.P., Eisenmann, J.C., Ribeiro, B., \& Aroso J. (2005). Maturity-associated variation in sport-specific skills of youth soccer players aged 13-15 years. Journal of Sports Sciences, 23, 515-522.

Malina, R., Pena, M.E., Eisenmann, J., Horta, L., Rodrigues, J., \& Miller, R. (2000). Height, mass and skeletal maturity of elite Portuguese soccer players aged 11-16 years. Journal of Sports Sciences, 18, 685-693.

Malý, T., Zahálka, F., Malá, L., \& Teplan, J., (2014). Profile, correlation and structure of speed in youth elite soccer players. Journal of Human Kinetics, 40(1), 149-159.

Manolopoulos, E., Papadopoulos, C., \& Kellis, E. (2006). Effects of combined strength and kick coordination training on soccer kick biomechanics in amateur players. Scandinavian Journal of Medicine and Science in Sports, $16(2), 102-110$.

Marfell-Jones, M., Olds, T., Stewart, A., \& Carter, L. (2006). International standards for anthropometric assessment. Potchefstroom: International Society for the Advancement of Kinanthropometry (ISAK).

Markovic, G., Dizdar, D., \& Jaric, S. (2006). Evaluation of tests of maximum kicking performance. Journal of Sports Medicine and Physical Fitness, 46(2), 215-220.

Masuda, K., Kikuhara, N., Demura, S., Katsuta, S., \& Yamanaka, K. (2005). Relationship between muscle strength in various isokinetic movements and kick performance among soccer players. Journal of Sports Medicine and Physical Fitness, 45(1), 44-52.

Mercé, J., González, L., Mayo, C., Pardo, A., \& Sorli, J. (2004). Evaluación de la condición física específica, en jugadores infantiles y cadetes de fútbol. [Assessment of specific physical condition in young elite soccer players. In Spanish.] Paper presented at the III Congreso de la Asociación Española de Ciencias del Deporte, Valencia.

Njororai, W.S. (2013). Analysis of goals scored in the 2010 World Cup soccer tournament held in South Africa. Journal of Physical Education and Sport, 13(1), 6-13.

Nunome, H., Lake, M., Georgakis, A., \& Stergioulas, L.K. (2006). Impact phase kinematics of instep kicking in soccer. Journal of Sports Sciences, 24(1), 11-22.

Oliva, S., Antonio, P., García Calvo, T., Marcos, L., Miguel, F., Sánchez Oliva, D., \& Amado Alonso, D. (2010). Análisis de la Importancia de la Utilización de la Pierna No Dominante en el Fútbol Profesional. [Analyzing the importance of using non-dominant leg in professional soccer players. In Spanish.] PubliCE Standard. Retrieved May 25, 2017 from: https://g-se.com/analisis-de-la-importancia-de-la-utilizacion-de-la-pierna-no-dominanteen-el-futbol-profesional-1234-sa-H57cfb271daa55 
Poulmedis, P., Rondoyannis, G., Mitsou, A., \& Tsarouchas, E. (1988). The influence of isokinetic muscle torque exerted in various speeds on soccer ball velocity. Journal of Orthopaedic and Sports Physical Therapy, 10(3), 93-96.

Rahnama, N., Lees, A., \& Bambaecichi, E. (2005). A comparison of muscle strength and flexibility between the preferred and non-preferred leg in English soccer players. Ergonomics, 48(11-14), 1568-1575.

Ramírez-Campillo, R., Gallardo, F., Henriquez-Olguín, C., Meylan, C.M., Martínez, C., Álvarez, C., Caniuqueo, A., Cadore, E.L., \& Izquierdo, M. (2015). Effect of vertical, horizontal, and combined plyometric training on explosive, balance, and endurance performance of young soccer players. Journal of Strength and Conditioning Research, 29(7), 1784-1795.

Ramírez-Campillo, R., Meylan, C., Álvarez, C., Henríquez-Olguín, C., Martínez, C., Cañas-Jamett, R., Andrade, D.C., \& Izquierdo, M. (2014). Effects of in-season low-volume high-intensity plyometric training on explosive actions and endurance of young soccer players. Journal of Strength and Conditioning Research, 28(5), 1335-1342.

Ré, A.H, Cattuzzo M.T, Santos F., \& Monteiro C. (2014). Anthropometric characteristics, field test scores and matchrelated technical performance in youth indoor soccer players with different playing status. International Journal of Performance Analysis in Sport, 14(2), 482-492.

Ré, A.H., Corrêa, U.C., \& Boehme, M.T. (2010). Anthropometric characteristics and motor skills in talent selection and development in indoor soccer. Perceptual and Motor Skills, 110(3), 916-930.

Reilly, T., Williams, A.M., Nevill, A., \& Franks, A. (2000). A multidisciplinary approach to talent identification in soccer. Journal of Sports Sciences, 18(9), 695-702.

Rodríguez-Lorenzo, L., Fernandez-del-Olmo, M., \& Martín-Acero, R. (2015). A critical review of the technique parameters and sample features of maximal kicking velocity in soccer. Strength and Conditioning Journal, 37(5), 26-39.

Rodríguez-Lorenzo, L., Fernandez-del-Olmo, M., Sánchez-Molina, J, \& Martín-Acero, R. (2016). The role of vertical jumps and anthropometric variables in maximal kicking ball velocities in elite soccer players. Journal of Human Kinetics, 50(3), 143-153.

Ruas, C., Brown, L., \& Pinto, R. (2015). Lower-extremity side-to-side strength asymmetry of professional soccer players according to playing position. Kinesiology, 47(2), 188-192.

Schmidt, R.A. (1988). Motor control and learning (pp. 105-116). Champaign, IL: Human Kinetics.

Shan, G. (2009). Influence of gender and experience on the maximal instep soccer kick. European Journal of Sport Science, 9(2), 107-114.

Sousa, P., Garganta, J., \& Garganta, R. (2003). Estatuto posicional, força explosiva dos membros inferiores e velocidade imprimida à bola no remate em Futebol. [Positional status, explosive strength and ball speed in kicking performance of young soccer players. In Portuguese.] Revista Portuguesa de Ciências do Desporto, 3(3), 27-35.

Tol, J.L., Slim, E., van Soest, A.J., \& van Dijk, C.N. (2002). The relationship of the kicking action in soccer and anterior ankle impingement syndrome: A biomechanical analysis. American Journal of Sports Medicine, 30(1), 45-50.

Velázquez, M., \& Jorge, J. (2012). Fiabilidad de la escala feedback correctivo en jugadores universitarios de fútbol soccer: estudios preliminares. [Corrective feedback scale reliability in college soccer players: Preliminary studies. In Spanish.] (Doctoral dissertation, Universidad Autónoma de Nuevo León). Mexico: Facultad De Organización Deportiva.

Wong, P.L., Chamari, K., Dellal, A., \& Wisloff, U. (2009). Relationship between anthropometric and physiological characteristics in youth soccer players. Journal of Strength and Conditioning Research, 23, 1204-1210.

Young, W.B., \& Rath, D.A. (2011). Enhancing foot velocity in football kicking: The role of strength training. Journal of Strength and Conditioning Research, 25(2), 561-566.

Submitted: January 9, 2017

Accepted: January 30, 2018

Published Online First: March 30, 2018

Correspondence to:

Rafael Martín-Acero

Departamento de Educación Física e Deportiva

Faculty of Sciences of Sport and Physical Education,

University of A Coruña

Av. Ernesto Che Guevara 121, Pazos-Liáns, 15179

Oleiros, A Coruña, Spain

Phone: +34 981167000

Fax: +34 981167048

E-mail: maracero@udc.es 